\title{
miR-410 enhanced hESC-derived pancreatic endoderm transplant to alleviate gestational diabetes mellitus
}

\author{
Yang $\mathrm{Mi}^{1}$, Na Guo', Tongqiang $\mathrm{He}^{1}$, Jing $\mathrm{Ji}^{1}$, Zhibin $\mathrm{Li}^{1}$ and Pu Huang ${ }^{2}$ \\ 'Obstetrical Department, Northwest Women's and Children's Hospital, 1616 Yanxiang Road, Xi'an, \\ Shanxi Province 710061, China \\ ${ }^{2}$ Department of Obstetrics and Gynecology, The First Affiliated Hospital of Xi'an Jiaotong University \\ College of Medicine, 277 Yanta West Road, Xi'an, Shanxi Province 710061, China
}

Correspondence should be addressed to $\mathrm{Y}$ Mi or P Huang

Emails

miyangmm@163.com or hp752000@163.com

\begin{abstract}
Gestational diabetes mellitus (GDM) is a condition commonly encountered during mid to late pregnancy with pathologic manifestations including hyperglycemia, hyperinsulinemia, insulin resistance, and fetal mal-development. The deficit and dysfunction of insulin secreting $\beta$-cells are signature symptoms for GDM. Pancreatic progenitors derived from human embryonic stem cells ( $\mathrm{hESCS}$ ) were shown to be able to effectively treat diabetes in mice. In this study, we first identified that microRNA-410 (miR-410) directly targets lactate dehydrogenase $A$ (LDHA), a gene selectively repressed in normal insulin secreting $\beta$-cells. hESCs that can be induced to express miR-410 hence keeping LDHA levels in check were then differentiated in vitro into pancreatic endoderm, followed by transplantation into $d b /+$ mouse model of GDM. The transplant greatly improved glucose metabolism and reproductive outcome of the pregnant females suffering from GDM. Our findings describe for the first time the method of combining miRNA with hESCs, providing proof of concept by employing genetically modified stem cell therapy for treating GDM.
\end{abstract}

\author{
Key Words \\ - microRNA-410 \\ - gestational diabetes mellitus \\ - human embryonic stem cell \\ - pancreatic endoderm \\ - C57BL/KsJ-Lep ${ }^{d b /+}$ mouse
}

Journal of Molecular Endocrinology (2015) 55, 219-229

\section{Introduction}

Gestational diabetes mellitus (GDM) is a disease resulting from insufficient insulin production during pregnancy (American Diabetes Association 2004). GDM patients are commonly diagnosed in the second trimester during pregnancy, usually showing no prior sign of diabetes. Approximately $3-5 \%$ of GDM patients also remain diabetic after pregnancy (Perkins et al. 2007). Besides maternal diabetic symptoms, GDM is also characterized by fetal mal-development (Gardosi \& Francis 2009). The mechanisms underlying GDM remain largely unexplored, although its origins are thought to be multifactorial involving both genetic and environmental factors (Cypryk et al. 2008). Mutations in the pancreatic $\beta$-cell KATP channel subunits Kir6.2 (KCNJ11) and SUR1 (ABCC8) were reported to cause diabetes mellitus and hyperinsulinism (Gloyn et al. 2006). High-saturated-fat diet was also shown to be able to induce glucose abnormalities among pregnant women (Bo et al. 2001). The C57BL/KsJ-Lep $p^{d b /+}$ (abbreviated as $d b /+$ ) mouse serves as a promising heterozygous mutant animal model closely

Published by Bioscientifica Ltd 
mimicking human GDM symptoms (Kaufmann et al. 1981). Prior to pregnancy, they exhibited largely normal glucose tolerance until late gestation (Ishizuka et al. 1999, Lambin et al. 2007). Fetal development was also defective with fetus weight at term increased by $5-8 \%$ (Lawrence et al. 1989, Yamashita et al. 2001).

Advances in stem cell technology have provided exciting development in diabetes treatment in animal models. Human embryonic stem cells (hESCs) were used to differentiate the pancreatic endoderm (PE) capable of treating diabetic mice (Kroon et al. 2008). Pagliuca et al. (2014) have also demonstrated in a very recent study that a large scale in vitro differentiation of human induced pluripotent stem cells (hiPSCs) to generate glucoseresponsive insulin-producing pancreatic $\beta$-cells. These cells were able to secret human insulin into the serum and ameliorate hyperglycemia after being transplanted in diabetic mice.

MicroRNAs (miRNAs or miRs) are 21-25 nucleotide long, small non-coding RNA molecules. miRNAs recognize specific and complementary sequences predominantly found in the 3'-UTR on target mRNAs, either repressing translation or degrading these mRNAs (Bartel 2004, 2009). In the development of diabetes, various miRNAs were found to play different functions and roles, and may serve as potential biomarkers (Higuchi et al. 2015, Seyhan 2015). miR-146b was reported to regulate retinal inflammation through suppressing adenosine deaminase- 2 in diabetes (Fulzele et al. 2015). miR-192, miR-194, miR-204, and miR-215 were found enriched in human kidney (Sun et al. 2004), where they target genes important for kidney development and function (Oghbaei et al. 2015). However, to the best of our knowledge, there has been no study on the potential involvement in GDM.

In this study, we first identified miR-410 as a direct regulator targeting human lactate dehydrogenase A (LDHA). LDHA is usually kept in low expression level in insulin-secreting $\beta$-cells (Sekine et al. 1994), and its overexpression affects glucose-induced insulin secretion (Ainscow et al. 2000). We then transduced miR-410 into hESCs using a Tet-on system. These miR-410 transduced hESCs were further differentiated to PE cells and transplanted into the $d b /+$ female mice. We then assessed the effect of the PE transplant on GDM symptoms and fetal development of the $d b /+$ female mice. The transplant greatly improved glucose metabolism and reproductive outcome of the pregnant $d b /+$ females. In our present study, we aimed to provide proof-of-concept and experimental methods of combining miRNA technique with stem cell therapy in treating GDM.

\section{Materials and methods}

\section{miRNA transfection}

Human shMIMIC inducible lentiviral miR-410 was purchased from GE Lifesciences (Pittsburgh, PA, USA: product ID: VSH6904-224634910) along with SMARTvector Inducible Non-targeting Control (product ID: VSC6572). Both miRNA and negative control were transduced into cell lines according to manufacturer's instructions.

\section{Luciferase reporter assay}

The putative miR-410 binding site at the 3'-UTR of LDHA was cloned downstream of a SV40 promoter-driven Gaussia luciferase (GLuc) reporter gene in a pEZX-MT05 vector (GeneCopoeia, Rockville, MD, USA). Mutant forms of the luciferase constructs were also generated using standard PCR-based overlap-extension protocols. For luciferase reporter assay, HeLa cells $\left(3 \times 10^{4}\right)$ were plated in a 24-well plate and then co-transfected with $400 \mathrm{ng}$ of either miR-410 or miR-control, $200 \mathrm{ng}$ of either WT luciferase constructs, using Lipofectamine 2000 (Invitrogen) according to manufacturer's instruction. Cells were collected $48 \mathrm{~h}$ after transfection and analyzed using the DualLuciferase Reporter Assay System (Promega). The pEZXMT05 vector also contains a secreted alkaline phosphatase (SeAP) reporter driven by a CMV promoter and served as the internal control to correct the differences in transfection and harvest efficiencies.

\section{Quantitative RT-PCR}

Total RNA was isolated from cells using the RNeasy MiniPrep Kit (Qiagen). One microgram of total RNA was reverse-transcribed with Superscript II First-Strand Synthesis Kit (Life Technologies) as recommended by the manufacturer. GAPDH mRNA levels were measured for normalization and all data were presented as relative expression.

\section{In vitro differentiation of hESCs}

H1 hESC lines were cultured using mTeSR1 medium (STEMCELL Technology, Vancouver, BC, Canada) on Matrigel (BD Biosciences, San Jose, CA, USA) coated culture plates and passaged as previously described (Ludwig et al. 2006). The four-stage differentiation protocol was carried out as previously described (Kroon et al. 2008).

In stage $1, \mathrm{H} 1 \mathrm{hESCs}$ were transitioned to definitive endoderm (DE). At 75\% confluency, H1 hESCs were

Published by Bioscientifica Ltd. 
washed briefly with $1 \times$ PBS, then cultured with RPMI medium containing $100 \mathrm{ng} / \mathrm{ml}$ activin supplemented with $0,0.2$, and $0.2 \%$ (v/v) FBS (Hyclone, Pittsburgh, PA, USA) on days 1-3 respectively. Wnt3a was added to day 1 medium at final concentration of $25 \mathrm{ng} / \mathrm{ml}$ to improve the transition.

In stage 2, DE was further differentiated to endoderm of the primitive gut (PG) tube. DE was cultured for 3 days with RPMI medium containing 2\% FBS and supplemented with $50 \mathrm{ng} / \mathrm{ml} \mathrm{FGF10} \mathrm{and} 20 \mathrm{ng} / \mathrm{ml}$ KAAD-cyclopamine (CYC).

In stage 3, PG cells were treated with $0.5 \mu \mathrm{M}$ retinoic acid (RA) together with $20 \mathrm{ng} / \mathrm{ml} \mathrm{CYC}$ and $50 \mathrm{ng} / \mathrm{ml}$ FGF10 in DMEM supplemented with 1\% B27 (Invitrogen) for 3 days to become PDX1-expressing posterior foregut (PF) endoderm cells.

In stage 4, PF cells were further transformed into pancreatic and endocrine lineages. During 3 days of culture, RA was removed from the medium and $1 \mu \mathrm{M}$ DAPT and $50 \mathrm{ng} / \mathrm{ml}$ exendin- 4 were added into the medium.

Cells after stage 4 were characterized by co-expression of PDX1, FOXA2, and SOX9, and were referred as PE.

\section{Immunofluorescence}

Cells were washed with $1 \times$ PBS for three times and fixed with $4 \%$ paraformaldehyde for $10 \mathrm{~min}$ at room temperature, followed by $10 \mathrm{~min}$ permeabilization with $0.2 \%$ Triton X-100 in PBS. The cells were blocked for $1 \mathrm{~h}$ with $5 \%$ goat serum and $2 \%$ BSA. Diluted primary antibodies were then added to the culture and incubated at $4{ }^{\circ} \mathrm{C}$ overnight. IgG isotype control mouse and rabbit polyclonal IgG (Abcam, Cambridge, MA, USA) were used as control for staining respectively. After four washes with $1 \times$ PBS, diluted secondary antibodies against specific primary antibodies were added and incubated in the dark at room temperature for $1 \mathrm{~h} .4^{\prime}$,6-Diamidino-2-phenylindole (DAPI, Invitrogen) was added 10 min before the final wash. Pictures were taken under fluorescent microscope. The primary antibodies used were OCT4 (mouse, 1:200 dilution, Santa Cruz), SOX9 (rabbit, 1:300 dilution, Santa Cruz), PDX1 (rabbit, 1:300 dilution, Santa Cruz), and FOXA2 (mouse, 1:200 dilution, Santa Cruz). The secondary antibodies used were Alex 488 (goat anti-mouse, 1:500, Life Technologies) and Alexa 568 (goat anti-rabbit, 1:500, Life Technologies).

\section{Western blotting}

Cell lysate was added into $20 \mu \mathrm{l} 2 \times$ sample loading buffer $(0.125 \mathrm{M}$ of $5 \mathrm{M}$ Tris- $\mathrm{HCl}$, Amresco, Framingham, MA,
USA; $20 \%$ glycerol, Usb; $4 \%$ of $10 \%$ SDS, Amresco; $1 \%$ $\beta$-mercaptoethanol, Amresco; and $0.2 \%$ of $0.05 \%(w / v)$ bromophenol blue, Sigma) and boiled for $5 \mathrm{~min}$ before loading. Proteins were separated by SDS-PAGE, transferred to Immobilon-P membrane (Millipore, Cambridge, MA, USA), and were probed with antibodies against LDHA and $\beta$-actin as indicated. All antibodies were purchased from Cell Signaling (Beverly, MA, USA). The results were visualized using ECL Kit (Abcam) and observed by GeneGnome machine (Syngene, Cambridge, UK).

\section{Glucose-stimulated insulin secretion}

Glucose-stimulated insulin secretion assay was performed according to previously established methods (Pagliuca et al. 2014). Briefly, PE clusters were washed with Krebs buffer and were then pre-incubated in $2 \mathrm{mM}$ glucose for $2 \mathrm{~h}$ to remove residual insulin. Clusters were then washed twice, incubated in $2 \mathrm{mM}$ glucose for $30 \mathrm{~min}$, and supernatant collected. Then clusters were washed again, incubated in $20 \mathrm{mM}$ glucose for another $30 \mathrm{~min}$, and supernatant collected. Clusters were then dispersed into single cells and cell number was counted. Supernatant samples containing secreted insulin were processed using the 1-2-3 UltraSensitive Mouse Insulin ELISA Kit (ALPCO Diagnostics, Salem, NH, USA).

\section{LDHA activity assay}

LDHA activity in hESC-derived PE clusters, in the presence or absence of doxycycline, was assayed using LDH Activity Assay Kit (Sigma) according to manufacturer's instructions.

\section{Animals and transplant of hESC-derived cells}

The care and use of animals in this study followed the guidelines and protocol approved by the Institutional Animal Care and Use Committee (IACUC) of The First Affiliated Hospital of Xi'an Jiaotong University. The IACUC members approved this study. All efforts were made to minimize the number of animals used and their suffering. Six-week-old mice were housed with fresh food and water, temperature $\left(22 \pm 2^{\circ} \mathrm{C}\right)$, humidity (40-60\%), and light ( $12 \mathrm{~h}$ light: $12 \mathrm{~h}$ darkness cycle) for 2 weeks. The rodent diet with $18 \%$ protein, $5 \%$ fat was provided (Harlan Teklad, Madison, WI, USA). Mice were arbitrarily assigned to one of four groups in a generalized randomized complete block design, with 20 mice/group: WT female mice receiving sham transplants and administered with doxycycline (WT sham +dox); $d b /+$ female

Published by Bioscientifica Ltd. 
mice receiving sham transplants and administered with doxycycline $(d b /+$ sham + dox $) ; d b /+$ females receiving PE transplants and administered with doxycycline $(d b /+\mathrm{PE}+$ dox); and $d b /+$ females receiving PE transplants but without doxycycline administer $(d b /+P E-d o x)$. Doxycycline was administered in drinking water at a concentration of $1 \mathrm{mg} / \mathrm{ml}$, supplemented with $1 \%$ (wt:vol) sucrose to mask the bitter taste. Mice were anesthetized with inhalable isoflurane and received transplants of $5 \times 10^{6}$ stage 4 hESC-derived PE cells under the left kidney capsule. After transplant all mice were treated with oral enrofloxacin (Bayer Animal Health) for 1 week $(100 \mathrm{mg} / \mathrm{ml}$ in drinking water).

\section{Body weight, blood glucose, and plasma insulin}

Body weight, blood glucose, and plasma insulin were measured prior to PE transplant (day 0), 4 weeks after transplant (day 28), gestation day 0 (GD0), GD10, and GD20. Body weight was measured on a top-loading balance (Accu-622; Fisher Scientific, Waltham, MA, USA), and nonfasting maternal blood samples were obtained via tail venipuncture to determine insulin and glucose levels. Blood glucose levels were determined by glucometer (Lifescan Surestep, Fisher Scientific). Plasma insulin levels were quantified by 1-2-3 UltraSensitive Mouse Insulin ELISA Kit (ALPCO Diagnostics), which detects both insulin 1 and 2 .

\section{Body composition and metabolic assessment}

Body composition (lean mass and fat content) was measured using a dual-energy X-ray absorptiometry method (Sabre Bone Densitometry, Norland Med, White Plains, WI, USA). Plasma leptin was assayed using mouse leptin ELISA Kits (Crystal Chem, Inc., Downers Grove, IL, USA) respectively. Plasma free fatty acids (FFAs) and triglycerides (TGs) were assessed using Wako NEFAC (Wako Chemicals USA, Inc., Richmond, VA, USA) and Free Glycerol Reagent (Sigma).

\section{Liver weight and liver TG content}

Mice were sacrificed to dissect and weigh liver tissue at the end of the 16-week experiment. Liver TG content was measured by homogenizing liver in chloroform/methanol (vol:vol, 2:1) and incubated at room temperature for $4 \mathrm{~h}$. The tissue lysate was then dried in air and re-suspended by $\mathrm{KOH}(3 \mathrm{M})$ for $1 \mathrm{~h}$ incubating at $70{ }^{\circ} \mathrm{C} . \mathrm{MgCl}_{2}$ was used to neutralize the lysate and TG assay was performed as previously described.

\section{Statistical analysis}

All data were analyzed using Prism Software version 5 (GraphPad, San Diego, CA, USA). Data are presented as mean \pm s.D. Comparisons of two groups were made by unpaired Student's $t$-test and one-way ANOVA. $P<0.05$ was considered as statistical significant.

\section{Results}

\section{miR-410 directly targets and down-regulates LDHA}

Using www.microrna.org as our resource, we identified miR-410 as a potential regulator of LDHA mRNA, with one binding site in the 3'-UTR of LDHA (NM_005566; Fig. 1A and Supplementary Figure 1, see section on supplementary data given at the end of this article). To confirm LDHA is a bona fide target of miR-410, luciferase reporter assay was performed, using sequences from original 3'-UTR on LDHA mRNA as well as a mutated version (Fig. 1B). As a result, luciferase activity was dramatically reduced by co-transfection of miR- 410 , to $<30 \%$ of non-targeting control (NC) transfected levels (Fig. 1C), indicating that the LDHA 3'-UTR was indeed a direct target of miR-410.

We next transfected miR-410 into HeLa cell lines and analyzed both mRNA and proteins levels of LDHA. Compared with miR-NC transfections, introducing miR410 significantly reduced mRNA levels of LDHA (Fig. 1D), suggesting regulation of LDHA by miR-410 occurred mainly through mRNA degradation. Using antibody against LDHA, we were able to detect that its protein levels were also significantly down-regulated in miR-410 transfected HeLa cells, but not in miR-NC transfected experiment (Fig. 1E). Taken together, the preceding results clearly demonstrated that miR-410 down-regulated both the mRNA and protein levels of LDHA.

\section{miR-410 suppresses LDHA expression in hESC-derived PE cells}

H1 hESC was first transduced with miR-410 in a Tet-on 3G induction system to become miR-H1 hESC line. Without doxycycline induction, miR-H1 can readily maintain their pluripotentcy and other typical characteristics of hESC (data not shown). Next, following previously established four-stage differentiation protocol (Kroon et al. 2008), we differentiated miR-H1 hESCs to PE (miR-PE) (see 'Materials and methods'). Using immunofluorescence, we were able to confirm the cells expressed appropriate markers throughout the differentiation. At the beginning of

Published by Bioscientifica Ltd 
A

$$
\begin{aligned}
& \text { miR-410 3'-uguccgguagACACAAUAUAa-5' } \\
& |||||||||| \mid \\
& \text { LDHA 3'-UTR 5'-auguuuaccgUGUGUUAUAUa-3' }
\end{aligned}
$$

B

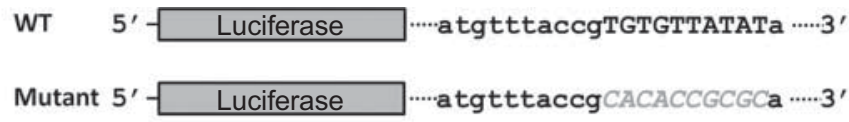

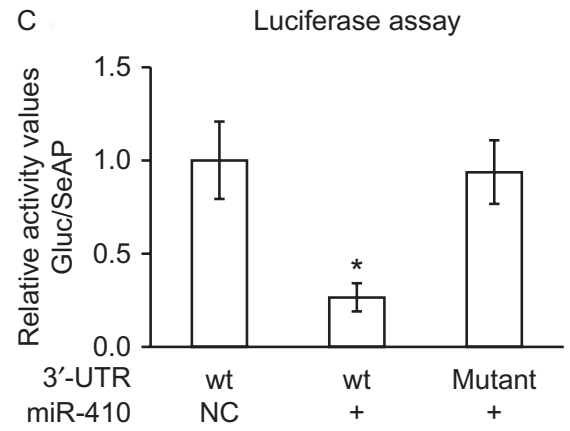

Figure 1

Sequence alignment of hsa-miR-410 and LDHA mRNA and confirmation of inhibitory function of hsa-miR-410 on LDHA using Luciferase Assay and HeLa cells. (A) Sequence alignment of hsa-miR-410 and LDHA mRNA.

(B) The construct for luciferase assay. (C) The relative activity of GLuc/SeAP

stage 1, H1 hESC expressed the pluripotency marker OCT4 (Niwa et al. 2000; Fig. 2A, top row). At stage 4, cells lost OCT4 and exhibited PDX1 expression (Fig. 2A, bottom row), a key pancreatic transcription factor (Jensen 2004). We further confirmed the cell lineage by staining the stage 4 hESC-derived cells with antibodies against FOXA2 and SOX9, another two signature transcription factors characterization of PE (Jensen 2004, Jorgensen et al. 2007), and they were both expressed in these cells (Fig. 2B). Next using quantitative RT-PCR, we quantified the relative expression levels of the previous marker genes, showing the differentiated cells had completely shut down OCT4 expression, and greatly increased mRNA levels of PDX1, FOXA2, and SOX9 (Fig. 2C). Therefore, the hESC-derived cells generated by our four-stage protocol closely matched committed PE in the embryo (Zorn \& Wells 2007).

Induced by doxycycline, miR-H1-ESCs showed significant decrease of LDHA mRNA levels compared to H1 hESCs without miR transduction. However, without doxycycline or miR-410, no significant difference in LDHA mRNA level was found compared with the control (Fig. 2D). Using western blot, we also found a decreased LDHA protein level when miR-PE cells were induced by doxycycline (Fig. 2E).

We next assessed whether the miR-PE exhibited ability to secret insulin when challenged with high levels of glucose. The miR-PE was subjected to glucose-stimulated insulin secretion assay, by sequential incubation with in luciferase assay. (D) Real-time GPCR results of LDHA expression in HeLa cells transfected with no miR, miR-410, and miR-NC. (E) Western blot results for LHDA protein in HeLa cells transfected with no miR, miR-410, and miR-NC. All values are given as mean, error bar represents S.E.M. ${ }^{*} P<0.05$.

2 and $20 \mathrm{mM}$ glucose, in the absence and presence of doxycycline to induce miR-410 expression (Fig. 2F). We found that when challenged with $20 \mathrm{mM}$ glucose, insulin secreted by the miR-PE was increased significantly compared with $2 \mathrm{mM}$ glucose, demonstrating the ability of the miR-PE to produce and secret insulin stimulated by glucose. Of note, insulin secretion of miR-PE in the presence of doxycycline was also markedly higher than that without induction, at both glucose levels (Fig. 2F, ${ }^{\#} P<0.05$ ). Further assay confirmed the drop in LDH activity upon doxycycline induction in the miR-PE, compared with the absence of doxycycline (Fig. 2G), which is consistent with previous reports that reduction in LDHA level increased glucose-induced insulin secretion in $\beta$-cells (Zhao \& Rutter 1998, Ainscow et al. 2000).

\section{miR-PE transplant alleviated hyperglycemia, hyperinsulinemia, and overweight in pregnant mice}

miR-PE was transplanted under the left kidney capsule of female $d b /+$ mice (see 'Materials and methods'). Their blood glucose levels, plasma insulin levels, and body weight were measured for all four experimental groups of female mice before PE transplant (day 0), 4 weeks after transplant (day 28), GD0, GD10, and GD20.

Blood glucose levels of all four experimental groups were almost the same prior to pregnancy (Fig. 3A). With the start of pregnancy, blood glucose levels of WT

Published by Bioscientifica Ltd 

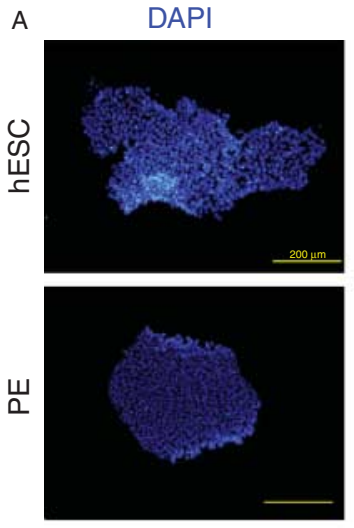

OCT4
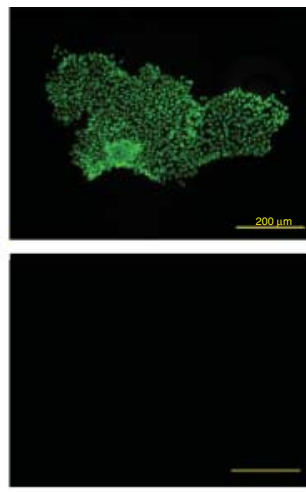

PDX1
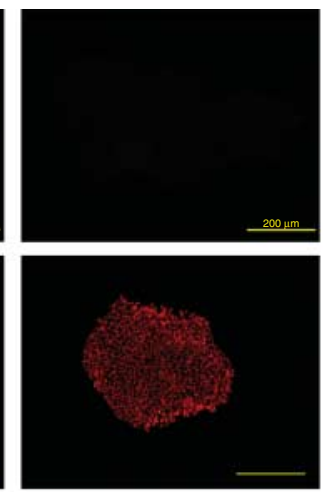

B

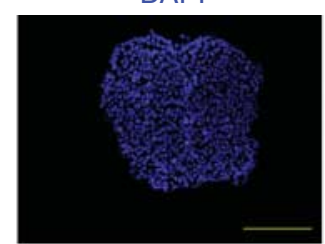

FOXA2

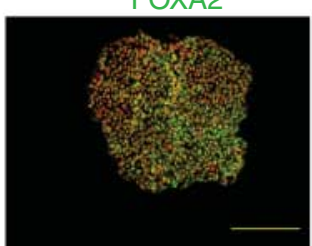

SOX9

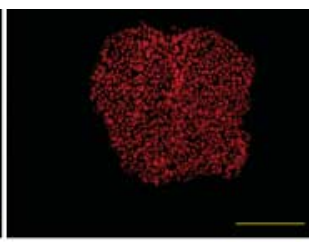

SOX9/FOXA2
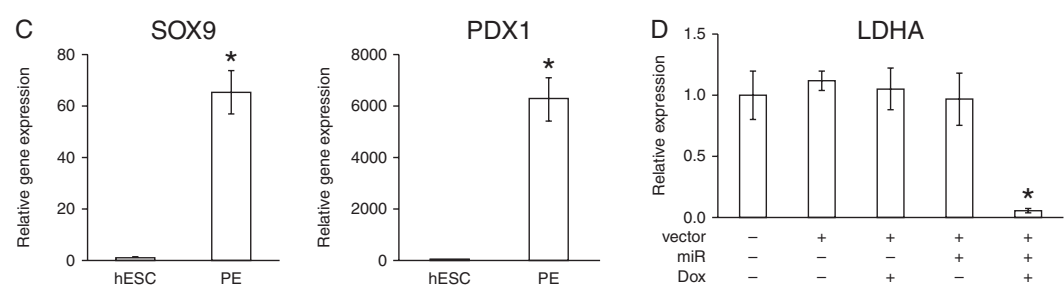

OCT4
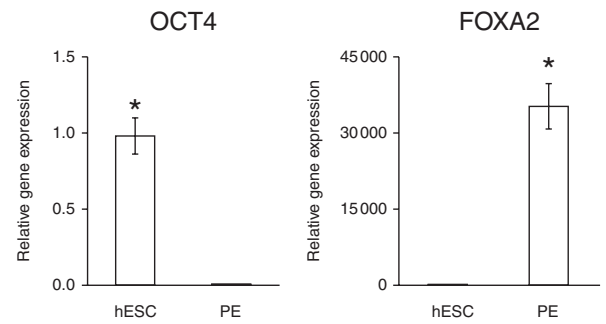

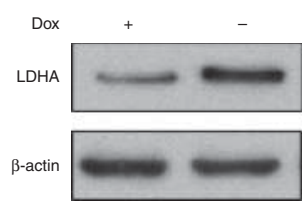

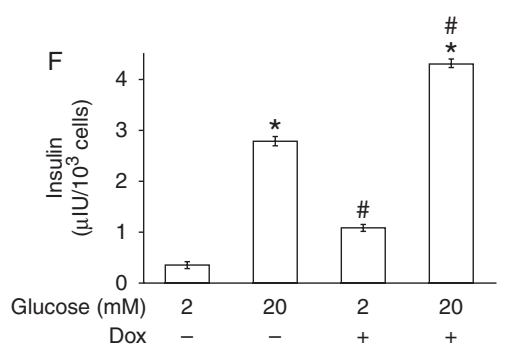

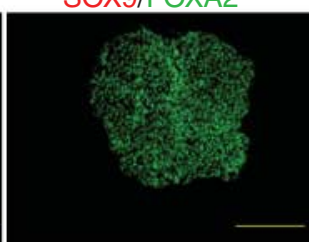

G

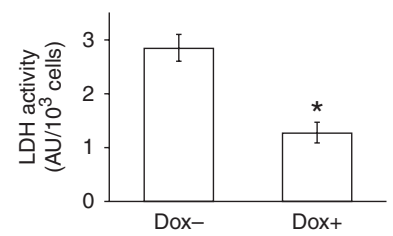

Figure 2

Characterization of hESC-derived PE and miR function. (A) miR-410-3p transfected H1-ESCs (miR-H1-ESC) were differentiated to pancreatic endoderm (PE) (see 'Materials and methods'). Cells of undifferentiated miRH1-ESC and differentiated PE (miR-PE) were subject to immunofluorescence analysis and stained for DAPI, OCT4, and PDX1. (B) miR-PE stained for DAPI, SOX9, and FOXA2. (C) Relative gene expression of OCT4, PDX1, FOXA2, and SOX9 from miR-hESCs and miR-PE. All values are given as mean, error bar represents S.E.M. ${ }^{*} P<0.05$ compared with undifferentiated $\mathrm{H} 1 \mathrm{hESC}$.

(D) Real-time qPCR results of LDHA expression in H1-ESC with and without miR-410-3p transfection and doxycycline induction. All values are given as

sham + dox females remained stable. In the meantime as expected, $d b /+$ sham + dox females showed a significant rise in blood glucose levels, presenting the typical GDM symptom of hyperglycemia during pregnancy. Whereas blood glucose levels of $d b /+\mathrm{PE}-$ dox females stayed at significantly lower levels throughout pregnancy than $d b /+$ sham + dox, indicating the alleviating effect on hyperglycemia by the miR-PE transplant. More importantly, in the $d b /+\mathrm{PE}+$ dox group, with doxycycline inducing the expression of miR-410, blood glucose level was as low as the WT sham+dox females, suggesting mean, error bar represents S.E.M. ${ }^{*} P<0.05$ compared with non-transduced H1 hESC. (E) Western blot analysis of LDHA in miR-PE cells with or without doxycycline induction. (F) ELISA measurements of secreted insulin from miR-PE clusters were sequentially incubated with 2 and $20 \mathrm{mM}$ glucose for 30 min respectively, in the presence or absence of doxycycline. ${ }^{\star} P<0.05$ compared with respective $2 \mathrm{mM}$ glucose incubation. ${ }^{\#} P<0.05$ compared with no doxycycline induction of the same glucose level. (G) LDH activity of miR-PE clusters were measured in the presence or absence of doxycycline. ${ }^{*} P<0.05$ compared with Dox - control.

down-regulation of LDHA by miR-410 in the PE transplant exhibited even more efficacy in treating hyperglycemia.

Plasma insulin levels in all four groups were also very similar before pregnancy (Fig. 3B). However $d b /+$ sham + dox group started producing more insulin after pregnancy, whereas no increase was observed in WT sham + dox and only mild increase in $d b /+\mathrm{PE}-$ dox groups at the same time point. This suggested the PE transplant relieved hyperinsulinemia during pregnancy. As expected, $d b /+$ $\mathrm{PE}+$ dox females exhibited same plasma insulin level as the WT sham+dox control, further demonstrating the

Published by Bioscientifica Ltd. 

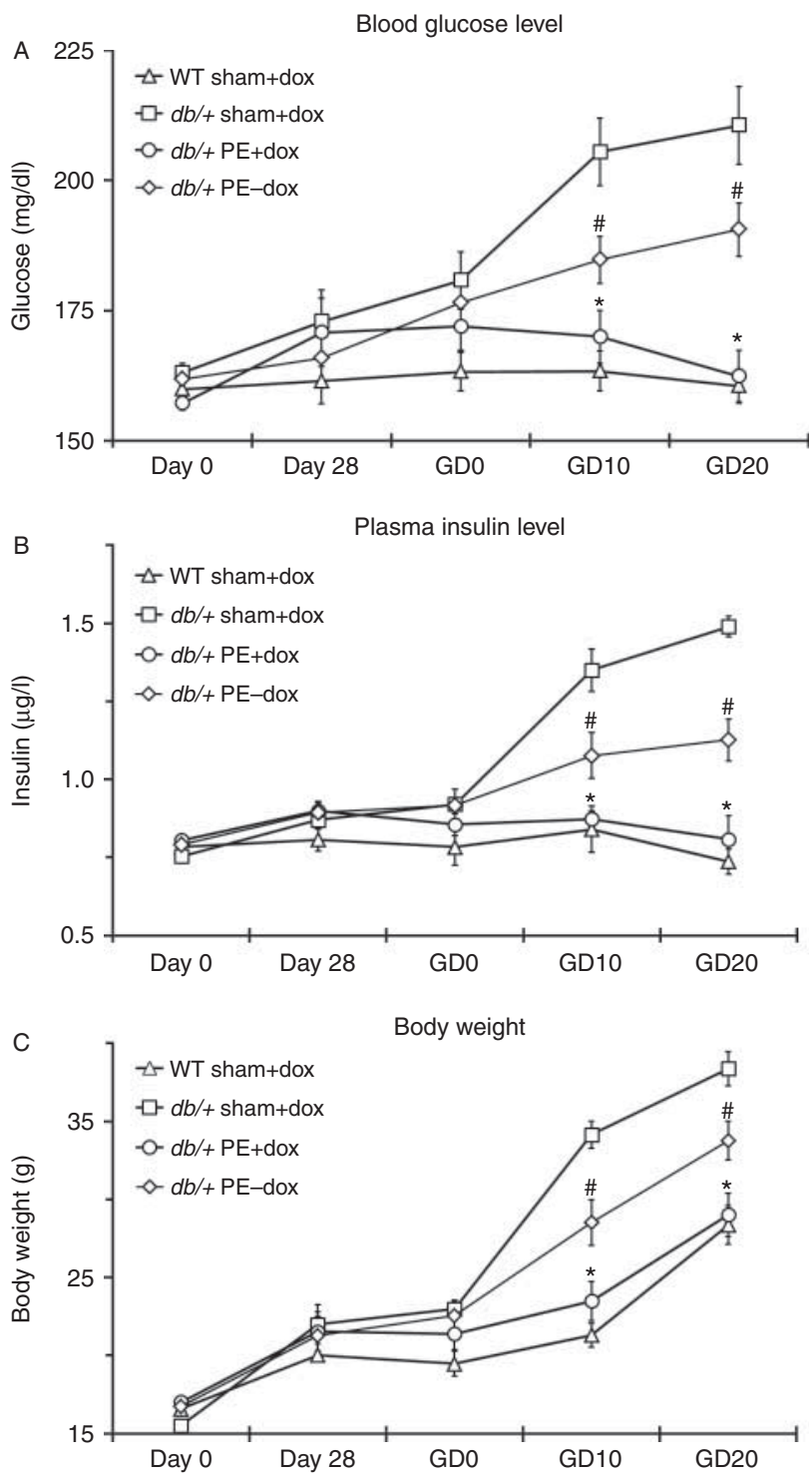

Figure 3

PE transplant alleviated GDM in pregnant mice. Blood glucose levels (A), plasma insulin levels (B), and maternal body weight (C) were measured prior to PE transplant (day 0), 4 weeks after transplant (day 28), and during pregnancy (GDO, 10, and 20) in WT sham + dox, $d b /+$ sham + dox, $d b /+P E+$ dox, and $d b /+P E-$ dox groups. ${ }^{*} P<0.05 d b /+P E+$ dox vs $d b /+P E-$ dox and $d b /+$ sham + dox at the same time point. ${ }^{\#} P<0.05 d b /+P E-$ dox vs with all the other three groups at the same time point.

extended efficacy of miR-410 expression in alleviating GDM hyperinsulinemia.

Finally, in Fig. 3C, body weight of WT sham + dox increased gradually during the entire span of the experiment. In a sharp comparison, $d b /+$ sham + dox females had much more rapid body weight gain during pregnancy, and $d b /+\mathrm{PE}-$ dox females exhibited slightly less weight gain in the same time span, showing the PE transplant was able to efficiently reduce GDM-resulted body weight gain. Again, the body weight of $d b /+\mathrm{PE}+$ dox group exhibited the same trend throughout the pregnancy as the WT sham +dox control, both significantly lower than either $d b /+$ sham + dox or $d b /+\mathrm{PE}-$ dox, which also suggested down-regulation of LDHA by miR-410 could completely nullify abnormal body weight gain during pregnancy as a result of GDM.

\section{miR-PE transplant maintains fat content, liver weight and TGs, plasma TGs and FFAs, and leptin at baseline levels}

We have so far demonstrated consistent results that in pregnant mice, miR-PE transplant that down-regulated LDHA exhibited significant effect in treating GDMrelated symptoms, with worst symptoms and maximum alleviating effect towards the end of pregnancy (GD20). Therefore, we next investigated, at GD20, the effect of miR-PE transplant at the organ and tissue development level, since studies have shown underlying correlation between liver weight and obesity in diabetes (Yang et al. 1997). We started with measuring fat content using WT sham + dox group as baseline control and $d b /+$ sham + dox as GDM control. There is no significant difference in fat content between $d b /+\mathrm{PE}+$ dox and baseline control, while $d b /+\mathrm{PE}-$ dox mice have significantly higher fat content than baseline albeit still lower than GDM control (Fig. 4A). This suggested that mice with miR-PE transplants can better control fat deposition than GDM mice, and achieve even better treatment with miR-410 induction hence LDHA down-regulation.

The liver is a major metabolic tissue for energy homeostasis such as synthesizing TGs de novo from glucose or FFAs obtained from diet and producing very LDL (VLDL) to transport to the circulatory system and eventually to other parts of the animal body (McDevitt et al. 2001, Strable $\&$ Ntambi 2010). In this context, liver weight and live TG of mice received miR-PE transplant were also maintained at only slightly higher level than WT sham + dox baseline control, whereas the measurement of $d b /+$ sham + dox mice increased remarkably higher than baseline (Fig. 4B and C). Similarly, inducing miR-410 by administering doxycycline after miR-PE transplant was able to further reduce both liver weight and TG to baseline level.

In the liver, FFAs are produced by the breakdown of TG obtained either from diet or cells like adipocytes as energy source of the body. With adequate plasma, FFAs and TGs from diet, liver synthesizes VLDLs and transports them to adipocytes for storing fat or muscle cells for producing energy. Whereas during fasting, plasma FFA and TG are insufficient to support energy consumption,

Published by Bioscientifica Ltd. 

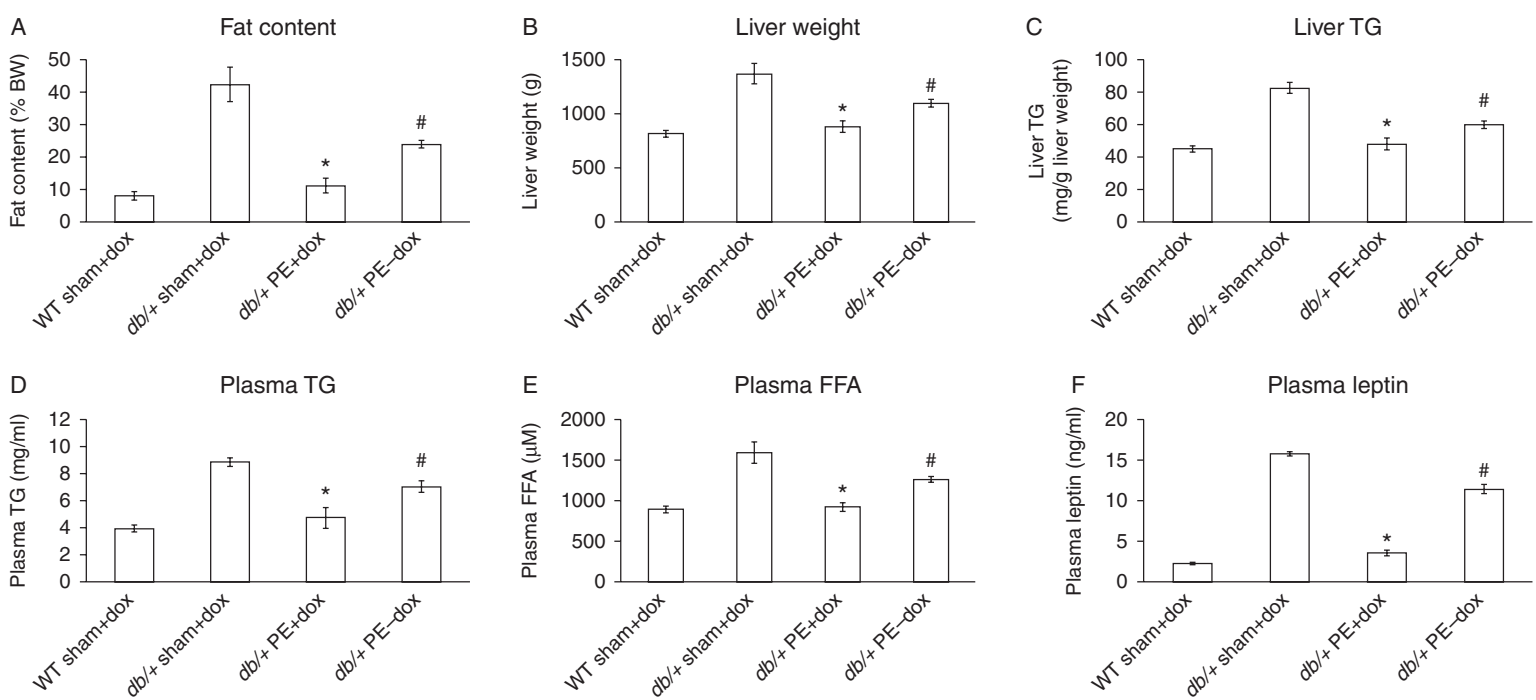

\section{Figure 4}

PE transplant alleviated diabetic symptoms in pregnant mice. Fat content (A), liver weight (B) and TG level (C), plasma TG (D), FFA (E), and leptin levels (F) were measured at GD20, in WT sham + dox, $d b /+$ sham + dox, $d b /+P E+$

liver then starts decomposing the stored fat. Elevated FFA influx is another factor causing TG accumulation in the liver, which is also related to diabetes and obesity (Adams et al. 2005). We next measured plasma FFA and TG in the four groups of female mice at GD20 (Fig. 4D and E), and found that both $d b /+$ sham + dox and $d b /+\mathrm{PE}-$ dox mice exhibited significantly increased levels of plasma FFA and TG after fasting, compared to WT sham+dox mice baseline. As expected, plasma FFA and TG in $d b /+\mathrm{PE}+$ dox mice were fully restored to the baseline level.

Finally, we assessed plasma leptin level. Leptin is a hormone produced by adipose tissue and functions to stimulate glucose uptake and control energy expenditure by facilitating fatty acid oxidation and preventing lipid accumulation through activating AMP kinase (Gil-Campos et al. 2004), serving as another indicator of diabetes and obesity in the GDM mice. Plasma leptin concentration after fasting in both $d b /+$ sham + dox and $d b /+P E-$ dox mice were significantly increased compared to WT sham + dox mice (Fig. 5F), while no significant difference from the baseline leptin level was found in $d b /+\mathrm{PE}+$ dox mice.

\section{miR-PE transplant improved reproductive outcome of GDM females}

One reported adverse effect of GDM is fetal mal-development, in that it worsened reproductive outcome in animal model with less fetuses (Siemelink et al. 2002, Buckley et al. dox, and $d b /+P E-$ dox groups. ${ }^{*} P<0.05 d b /+P E+$ dox vs $d b /+P E-$ dox and $d b /+$ sham + dox. ${ }^{\#} P<0.05 d b /+P E-$ dox vs with all the other three groups.

2005). Particularly in $d b /+$ GDM mouse model, fetus weight at term was reported to increase by 5-8\% (Lawrence et al. 1989, Yamashita et al. 2001). This has prompted us to investigate whether our miR-PE transplant was able to improve fetal development of GDM female mice.

Total litter number at birth was counted from equal number of dam $(n=20)$ from WT sham + dox (151 litters), $d b /+$ sham $+\operatorname{dox}$ (83 litters), $d b /+P E+\operatorname{dox}$ (145 litters), and $d b /+\mathrm{PE}-$ dox (104 litters) dam groups and plotted in Fig. 5A. Moreover, the mean body weight of litters at birth was also recorded, and we observed that the mean litter weight from $d b /+$ sham + dox and $d b /+\mathrm{PE}-$ dox dam groups were significantly higher than that of WT sham + dox, whereas $d b /+\mathrm{PE}+$ dox female mice gave birth to litters with nearly the same mean body weight as the WT sham + dox dam (Fig. 5B). Survival rate of litters was also monitored daily during the first 2 weeks (Fig. 5C). Litters born by $d b /+$ sham + dox and $d b /+P E-$ dox dams had significantly higher mortality rate (Fig. 5C, black and blue curves respectively) than the other two groups (red and green curves). These results clearly indicated miR-PE transplant was able to increase the offspring number and the reproductive outcome of GDM female mice.

\section{Discussion}

GDM affects nearly $10 \%$ of all pregnancies, and females suffering GDM have increased life-long risk of type 2

Published by Bioscientifica Ltd 
A

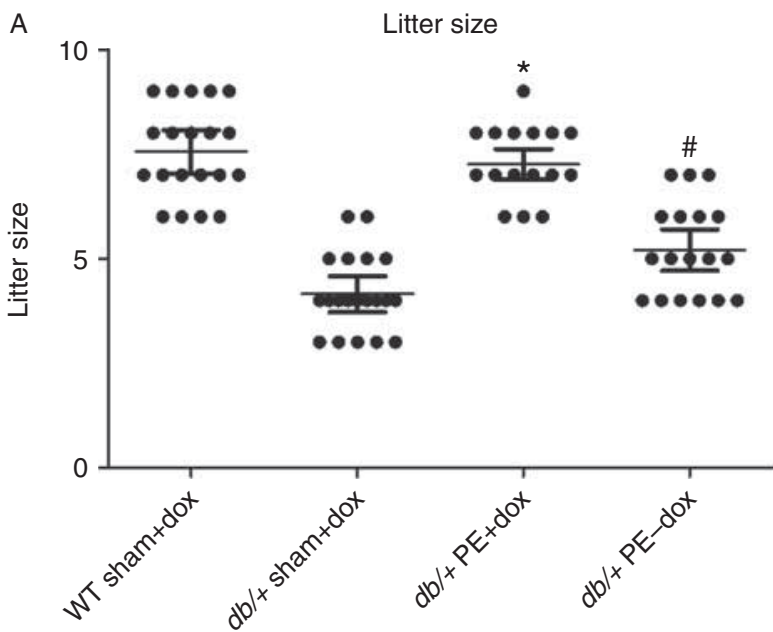

B

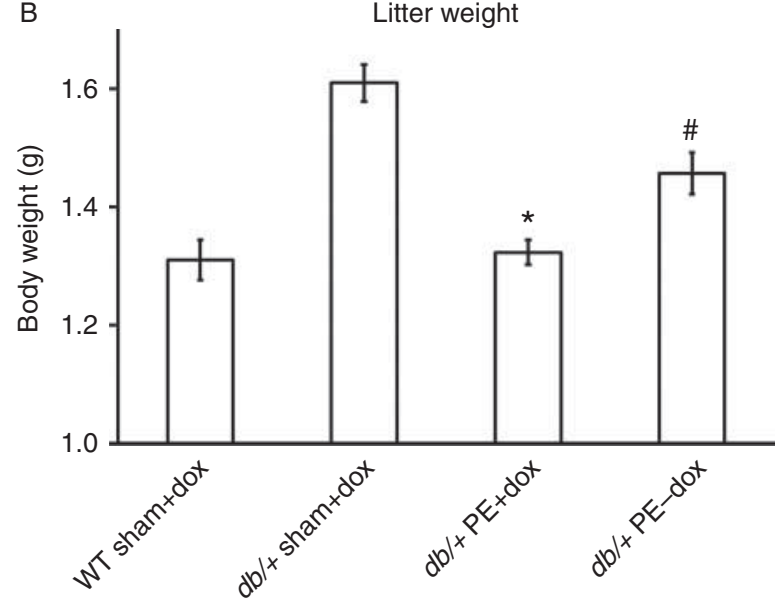

C

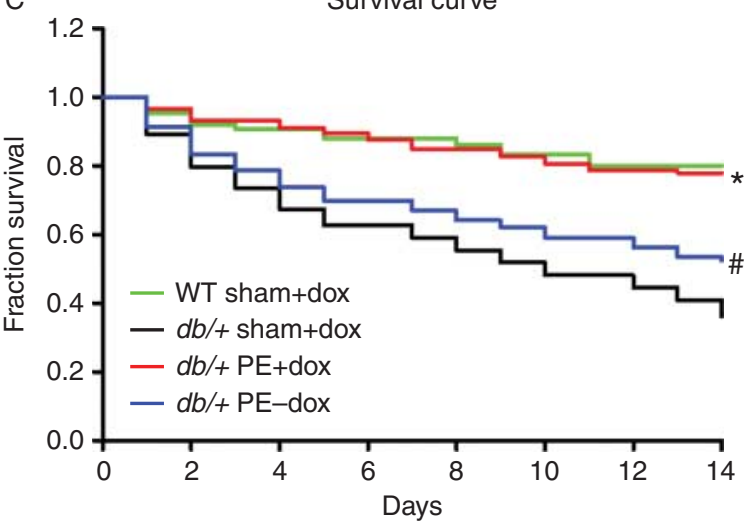

Figure 5

PE transplant improved reproductive outcome of GDM female mice. Size (A) and body weight (B) of litters at birth was counted for each female mouse from WT sham + dox, $d b /+$ sham + dox, $d b /+P E+$ dox, and $d b /+$ $P E$ - dox dam groups $(n=20)$. (C) Kaplan-Meier survival curves of litters from four different groups of dams. Litters were housed with their respective dam from birth till day 14 , and were monitored daily and dead litters were recorded and removed from the cage. ${ }^{*} P<0.05 \mathrm{db} /+\mathrm{PE}+\mathrm{dox}$ vs $d b /+P E-d o x$ and $d b /+$ sham + dox. ${ }^{\#} P<0.05 d b /+P E-$ dox vs with all the other three groups.

diabetes (American Diabetes Association 2004). Therefore, studies to understand the underlying mechanism of GDM are of great clinical value. Stem cell therapy utilizing hESCs and hiPSCs have been widely used in animal models and were shown to successfully alleviating diabetic symptoms (Kroon et al. 2008, Pagliuca et al. 2014). These studies have made possible several clinical trials showing promising outcomes in treating diabetes (Viswanathan \& Sarang 2013). However, few studies were conducted on GDM in particular, which still focused on dietary intervention method, instead of stem cell therapy or molecular and genetic approaches hence yet provided fruitful results (Landon et al. 2009). Reasons for the lack of success could be attributed to insufficient experimental data on animal models.

Given the relative similar cause and symptoms between type 2 diabetes and GDM, we designed our study by employing stem cell therapy that has previously proven successful, and implementing a miRNA Tet-on inducible system that specifically targets LDHA, whose overexpression affects glucose-induced insulin secretion in $\beta$-cells, therefore, is usually kept in check (Sekine et al. 1994). Indeed, we observed an interesting result, that glucosestimulated insulin secretion of the miR-PE in the presence of doxycycline was markedly higher than that without induction (Fig. 2F). This particular result confirmed previous reports that down-regulation of LDHA level led to elevated insulin secretion upon glucose stimulation in $\beta$-cells (Zhao \& Rutter 1998, Ainscow et al. 2000). Moreover, in a later part of our study, although in general the PE transplant exhibited alleviation of GDM symptoms, we have consistently observed significant better effects in female mice receiving both miR-PE transplant and doxycycline than those receiving only the PE transplant. We speculate that this improvement in efficacy could be explained by the reduction of LDHA levels in the miR-PE (Fig. 2E), which indicated a potential involvement of LDHA in GDM. There has been no report on the role of LDH in GDM, except that LDHB was found to be down-regulated in adipose tissue from GDM women (Oliva et al. 2013). This and our results suggest a possible distinct regulation of LDHs as well as their roles in contributing to GDM in mammals.

Although sharing the usual suspect of diabetic symptoms, such as hyperglycemia, hyperinsulinemia, overweight, elevated fat content, liver and plasma TG, FFA and leptin levels, GDM is distinct from type 2 diabetes in that GDM also affects reproductive outcome and fetal development (Lawrence et al. 1989, Ishizuka et al. 1999, Yamashita et al. 2001, Siemelink et al. 2002, Buckley et al. 2005). Results from our study were consistent with reports on the same $d b /+$ GDM mouse model, with $\sim 10 \%$ increase in body

Published by Bioscientifica Ltd 
weight from litters born by GDM dam, whereas miR-PE transplated GDM females gave birth to litters with body weight comparable to those from WT dam. Therefore, our results supported the potential mechanism where genetic disposition of GDM in pregnant females not only perturbed metabolism during pregnancy, but also adversely affected development and/or metabolism of the fetus, hence abnormally increasing their body weight. Moreover, during the first 2 weeks after birth, litters from $d b /+$ mice exhibited severely reduced survival rate compared to those from the other groups of dams (Fig. 5), suggesting early development of offsprings was also affected by GDM, and more importantly the miR-PE transplant efficient rescued this early developmental defect, which further demonstrated the efficacy of our approach by combined methods.

To summarize, our current study has provided the new method of treating GDM in the $d b /+$ transgenic mouse model, by combining miRNA technique with stem cell therapy. Our results have demonstrated that the miR-PE transplant alleviated hyperglycemia and hyperinsulinemia in pregnant female mice, and significantly improved their reproductive outcome, as well as early offspring development. Moreover, by inducing the expression of miR-410 to repress LDHA levels in the miR-PE, the effect of the transplant in treating GDM was improved even further. Therefore, our study has provided evidence supporting the potential therapeutic values of not only stem cell therapy, but also incorporation of molecular and genetic modifications to achieve better clinical efficacy in treating GDM in human patients.

\section{Supplementary data}

This is linked to the online version of the paper at http://dx.doi.org/10.1530/ JME-15-0100.

\section{Declaration of interest}

The authors declare that there is no conflict of interest that could be perceived as prejudicing the impartiality of the research reported.

\section{Funding}

This work was supported by Shanxi's Science and Technology Research Program (2008K14-04).

\section{References}

Adams LA, Angulo P \& Lindor KD 2005 Nonalcoholic fatty liver disease. CMAJ: Canadian Medical Association Journal 172 899-905. (doi:10.1503/ cmaj.045232)
Ainscow EK, Zhao C \& Rutter GA 2000 Acute overexpression of lactate dehydrogenase-A perturbs $\beta$-cell mitochondrial metabolism and insulin secretion. Diabetes 49 1149-1155. (doi:10.2337/diabetes. 49.7.1149)

American Diabetes Association 2004 Gestational diabetes mellitus. Diabetes Care 27 (Suppl 1) S88-S90.

Bartel DP 2004 MicroRNAs: genomics, biogenesis, mechanism, and function. Cell 116 281-297. (doi:10.1016/S0092-8674(04)00045-5)

Bartel DP 2009 MicroRNAs: target recognition and regulatory functions. Cell 136 215-233. (doi:10.1016/j.cell.2009.01.002)

Bo S, Menato G, Lezo A, Signorile A, Bardelli C, De Michieli F, Massobrio M \& Pagano G 2001 Dietary fat and gestational hyperglycaemia. Diabetologia 44 972-978. (doi:10.1007/s001250100590)

Buckley AJ, Keseru B, Briody J, Thompson M, Ozanne SE \& Thompson CH 2005 Altered body composition and metabolism in the male offspring of high fat-fed rats. Metabolism 54 500-507. (doi:10.1016/j.metabol. 2004.11.003)

Cypryk K, Szymczak W, Czupryniak L, Sobczak M \& Lewinski A 2008 Gestational diabetes mellitus - an analysis of risk factors. Endokrynologia Polska 59 393-397.

Fulzele S, El-Sherbini A, Ahmad S, Sangani R, Matragoon S, El-Remessy A, Radhakrishnan R \& Liou GI 2015 MicroRNA-146b-3p regulates retinal inflammation by suppressing adenosine deaminase- 2 in diabetes. BioMed Research International 2015 846501. (doi:10.1155/2015/846501)

Gardosi J \& Francis A 2009 A customized standard to assess fetal growth in a US population. American Journal of Obstetrics and Gynecology 25 e21-e27.

Gil-Campos M, Canete R \& Gil A 2004 Hormones regulating lipid metabolism and plasma lipids in childhood obesity. International Journal of Obesity and Related Metabolic Disorders 28 (Suppl 3) S75-S80. (doi:10.1038/sj.ijo.0802806)

Gloyn AL, Siddiqui J \& Ellard S 2006 Mutations in the genes encoding the pancreatic $\beta$-cell KATP channel subunits Kir6.2 (KCNJ11) and SUR1 (ABCC8) in diabetes mellitus and hyperinsulinism. Human Mutation 27 220-231. (doi:10.1002/humu.20292)

Higuchi C, Nakatsuka A, Eguchi J, Teshigawara S, Kanzaki M, Katayama A Yamaguchi S, Takahashi N, Murakami K, Ogawa D et al. 2015 Identification of circulating miR-101, miR-375 and miR-802 as biomarkers for type 2 diabetes. Metabolism 64 489-497. (doi:10.1016/ j.metabol.2014.12.003)

Ishizuka T, Klepcyk P, Liu S, Panko L, Liu S, Gibbs EM \& Friedman JE 1999 Effects of overexpression of human GLUT4 gene on maternal diabetes and fetal growth in spontaneous gestational diabetic C57BLKS/J Lepr(db/+) mice. Diabetes 48 1061-1069. (doi:10.2337/diabetes.48.5.1061)

Jensen J 2004 Gene regulatory factors in pancreatic development. Developmental Dynamics 229 176-200. (doi:10.1002/dvdy.10460)

Jorgensen MC, Ahnfelt-Ronne J, Hald J, Madsen OD, Serup P \& Hecksher-Sorensen J 2007 An illustrated review of early pancreas development in the mouse. Endocrine Reviews 28 685-705. (doi:10.1210/er.2007-0016)

Kaufmann RC, Amankwah KS, Dunaway G, Maroun L, Arbuthnot J \& Roddick JW Jr 1981 An animal model of gestational diabetes. American Journal of Obstetrics and Gynecology 141 479-482.

Kroon E, Martinson LA, Kadoya K, Bang AG, Kelly OG, Eliazer S, Young H, Richardson M, Smart NG, Cunningham J et al. 2008 Pancreatic endoderm derived from human embryonic stem cells generates glucose-responsive insulin-secreting cells in vivo. Nature Biotechnology 26 443-452. (doi:10.1038/nbt1393)

Lambin S, van Bree R, Caluwaerts S, Vercruysse L, Vergote I \& Verhaeghe J 2007 Adipose tissue in offspring of Lepr $(\mathrm{db} /+)$ mice: early-life environment vs. genotype. American Journal of Physiology. Endocrinology and Metabolism 292 E262-E271. (doi:10.1152/ajpendo.00308.2006)

Landon MB, Spong CY, Thom E, Carpenter MW, Ramin SM, Casey B, Wapner RJ, Varner MW, Rouse DJ, Thorp JM Jr et al. 2009 A multicenter, randomized trial of treatment for mild gestational diabetes. New England Journal of Medicine 361 1339-1348. (doi:10.1056/ NEJMoa0902430) 
Lawrence S, Warshaw J \& Nielsen HC 1989 Delayed lung maturation in the macrosomic offspring of genetically determined diabetic $(\mathrm{db} /+)$ mice. Pediatric Research 25 173-179. (doi:10.1203/00006450-19890200000019)

Ludwig TE, Bergendahl V, Levenstein ME, Yu J, Probasco MD \& Thomson JA 2006 Feeder-independent culture of human embryonic stem cells. Nature Methods 3 637-646. (doi:10.1038/nmeth902)

McDevitt RM, Bott SJ, Harding M, Coward WA, Bluck LJ \& Prentice AM 2001 De novo lipogenesis during controlled overfeeding with sucrose or glucose in lean and obese women. American Journal of Clinical Nutrition 74 737-746.

Niwa H, Miyazaki J \& Smith AG 2000 Quantitative expression of Oct-3/4 defines differentiation, dedifferentiation or self-renewal of ES cells. Nature Genetics 24 372-376. (doi:10.1038/74199)

Oghbaei H, Ahmadi Asl N, Sheikhzadeh F, Alipour MR \& Khamaneh AM 2015 The effect of regular moderate exercise on miRNA-192 expression changes in kidney of streptozotocin-induced diabetic male rats. Advanced Pharmaceutical Bulletin 5 127-132.

Oliva K, Barker G, Rice GE, Bailey MJ \& Lappas M 2013 2D-DIGE to identify proteins associated with gestational diabetes in omental adipose tissue. Journal of Endocrinology 218 165-178. (doi:10.1530/JOE-13-0010)

Pagliuca FW, Millman JR, Gurtler M, Segel M, Van Dervort A, Ryu JH, Peterson QP, Greiner D \& Melton DA 2014 Generation of functional human pancreatic $\beta$ cells in vitro. Cell 159 428-439. (doi:10.1016/j.cell. 2014.09.040)

Perkins JM, Dunn JP \& Jagasia SM 2007 Perspectives in gestational diabetes mellitus: a review of screening, diagnosis, and treatment. Clinical Diabetes 25 57-62. (doi:10.2337/diaclin.25.2.57)

Sekine N, Cirulli V, Regazzi R, Brown LJ, Gine E, Tamarit-Rodriguez J, Girotti M, Marie S, MacDonald MJ, Wollheim CB et al. 1994 Low lactate dehydrogenase and high mitochondrial glycerol phosphate dehydrogenase in pancreatic $\beta$-cells. Potential role in nutrient sensing. Journal of Biological Chemistry 269 4895-4902.

Seyhan AA 2015 microRNAs with different functions and roles in disease development and as potential biomarkers of diabetes: progress and challenges. Molecular BioSystems.

Siemelink M, Verhoef A, Dormans JA, Span PN \& Piersma AH 2002 Dietary fatty acid composition during pregnancy and lactation in the rat programs growth and glucose metabolism in the offspring. Diabetologia 45 1397-1403. (doi:10.1007/s00125-002-0918-2)

Strable MS \& Ntambi JM 2010 Genetic control of de novo lipogenesis: role in diet-induced obesity. Critical Reviews in Biochemistry and Molecular Biology 45 199-214. (doi:10.3109/10409231003667500)

Sun Y, Koo S, White N, Peralta E, Esau C, Dean NM \& Perera RJ 2004 Development of a micro-array to detect human and mouse microRNAs and characterization of expression in human organs. Nucleic Acids Research 32 e188. (doi:10.1093/nar/gnh186)

Viswanathan C \& Sarang S 2013 Status of stem cell based clinical trials in the treatment for diabetes. Current Diabetes Reviews 9 429-436. (doi:10.2174/15733998113096660082)

Yamashita H, Shao J, Ishizuka T, Klepcyk PJ, Muhlenkamp P, Qiao L, Hoggard $\mathrm{N} \&$ Friedman JE 2001 Leptin administration prevents spontaneous gestational diabetes in heterozygous Lepr $(\mathrm{db} /+)$ mice: effects on placental leptin and fetal growth. Endocrinology 142 2888-2897.

Yang SQ, Lin HZ, Lane MD, Clemens M \& Diehl AM 1997 Obesity increases sensitivity to endotoxin liver injury: implications for the pathogenesis of steatohepatitis. PNAS 94 2557-2562. (doi:10.1073/pnas.94.6.2557)

Zhao C \& Rutter GA 1998 Overexpression of lactate dehydrogenase A attenuates glucose-induced insulin secretion in stable MIN-6 $\beta$-cell lines. FEBS Letters 430 213-216. (doi:10.1016/S0014-5793(98)00600-0)

Zorn AM \& Wells JM 2007 Molecular basis of vertebrate endoderm development. International Review of Cytology 259 49-111.

Received in final form 22 August 2015

Accepted 24 August 2015

Accepted Preprint published online 25 August 2015
(C) 2015 Society for Endocrinology Printed in Great Britain 